\title{
THE USNO ASTROMETRIC OPTICAL INTERFEROMETER
}

\author{
D.J. HUTTER, K.J. JOHNSTON \\ United States Naval Observatory \\ Washington, DC 20392-5420
}

AND

D. MOZURKEWICH

Remote Sensing Division, Naval Research Laboratory

Washington, DC 20375

\begin{abstract}
The U.S. Naval Observatory Astrometric Optical Interferometer (AOI) began operation on Anderson Mesa, near Flagstaff, Arizona, in the autumn of 1994. The AOI incorporates four siderostats that are located in a $\mathrm{Y}$-shaped configuration, and features a full-array laser metrology system to monitor baseline motion. The AOI incorporates state-of-the-art delay lines and a real-time fringe-tracking system. The AOI will have a limiting visual magnitude of 10 , under typical observing conditions, and will produce star positions accurate to a few milliarcseconds (mas). With a planned operational lifetime of several decades, this instrument will be capable of maintaining the optical reference frame by improving the proper motions of thousands of the brighter HIPPARCOS stars through repeated observations.
\end{abstract}

\section{Introduction}

The U.S. Naval Observatory Astrometric Optical Interferometer (AOI) is the dedicated astrometric subarray of the new Navy Prototype Optical Interferometer (NPOI) at Lowell Observatory, which is being built in collaboration with the Naval Research Laboratory. The Naval Research Laboratory is constructing the imaging subarray of the NPOI, the "Big Optical Array" (BOA; Armstrong 1994).

The AOI was built using the experience gained from the Mark III Interferometer on Mt. Wilson, CA, which demonstrated "proof of concept" of wide-angle astrometry by a long baseline optical interferometer. The Mark III is a broadband phase-tracking optical interferometer, which was operational from late 1986 through 1992 (Shao et al. 1988). The Mark III is capable of single baseline measurements on either a north-south or east-south baseline, each of about $12 \mathrm{~m}$ in length. The Mark III incorporates evacuated delay lines with automated high speed servo systems, allowing the detection and tracking of white-light fringes 
in real time. Measurements at two different wavelengths are used to correct the measured delays for refractive index fluctuations in the turbulent atmosphere.

Measurements of stellar positions by the Mark III in a subset of the FK5 catalog, made at several epochs over a four-year period, have uncertainties of 10 to 20 mas (Hummel et al. 1994). The accuracy of the measurements is limited by systematic errors largely due to unmonitored changes in the baseline coordinates. These errors are due to mechanical and thermal motions within the instrument, principally in the siderostats. Therefore, in the design of the AOI, major consideration was given to an extensive baseline metrology system. This system will monitor fully the motions of the baselines in three dimensions with respect to a reference system fixed to the Earth's crust. The AOI also includes new multi-way beam combination and fringe detection techniques, along with an increase in the number of siderostats from two to four. These improvements allow simultaneous observations on six baselines (of lengths between $18 \mathrm{~m}$ and $35 \mathrm{~m}$, three of which are geometrically independent). Such observations will permit the total separation of the baseline components and star position corrections in the astrometric solutions. The addition of a dispersed fringe detection technique allows unambiguous central fringe identification, and better correction for atmospherically induced delay fluctuations. Additionally, the increased aperture, state-of-the-art delay lines, and greater detector sensitivity will permit the AOI to measure the positions of some of the brighter stellar radio sources, whose positions have been tied to the radio reference frame defined by extragalactic sources.

The combination of these improvements will permit the AOI to produce highly accurate catalogs of star positions on a nearly inertial frame. The initial goal of the AOI is to establish, by mid 1997, a catalog of the order of one thousand stars with statistical and systematic errors in the range of 1 to 3 mas. This catalog will be more than an order of magnitude more precise than the FK5 and of the same order as expected from space-based systems. With an anticipated operational lifetime of decades, the AOI will significantly improve the measured proper motions of these (and additional) stars. Stellar positions measured over a period of ten years will have proper motions known to a few $100 \mu \mathrm{as} / \mathrm{year}$. Position measurements repeated at regular intervals will also allow unambiguous separation of binary motion from proper motion, an accomplishment that might be difficult to achieve from space-based observations that are likely to be repeated only at intervals of decades.

Thus, the AOI, with a capability of milliarcsecond astrometry and a planned operational lifetime of several decades, will be capable of maintaining the optical reference frame by improving the proper motions of thousands of the brighter HIPPARCOS stars through repeated observations. 


\section{Description of the Instrument}

The light-collecting apertures of the AOI consist of four siderostats located in a Y-shaped array. Starlight collected by the siderostats is directed into the "feed system" vacuum pipes that carry the light to the center of the array. Additional mirrors at the array center redirect the light along the vacuum lines to the optical laboratory. The laboratory contains the delay lines, beam-combining optics, fringe detection system, and the star-tracking sensors. All interferometer systems are remotely controlled from a separate operations facility.

\subsection{SIDEROSTATS}

The siderostats are housed in roll-off-roof enclosures, which will be climatecontrolled to approximate average nighttime temperatures. The siderostats consist of permanently mounted, high-precision, two-axis alt-az mountings which each support a flat mirror of $50-\mathrm{cm}$ clear aperture. The siderostats are driven in each axis by a novel drive system consisting of in-line dual harmonic drive gear reduction units driven by stepper motors. The yoke and mirror cell of each siderostat include provisions for accurately intersecting the azimuth and elevation axes, and for placing the mirror surface on the elevation axis (both to within a few microns).

A wide field-of-view star acquisition capability is also incorporated via a CCD camera located near each siderostat. A two-axis gimbal-mounted flat mirror, with piezo-electric actuators, is located in the stellar light path near each siderostat. These mirrors are steered by the star-tracking sensors to provide the small angle, high speed tip-tilt correction necessary to compensate for random image motion due to atmosphere turbulence.

Provision is made within the siderostat enclosures for the addition of a $35-\mathrm{cm}$ aperture afocal beam compressor next to each siderostat. When the beam compressors are installed in 1995, the limiting sensitivity of the AOI will be increased from $\mathrm{V}=8.5$ (for the current aperture of $12.5 \mathrm{~cm}$, set by the feed system optics) to $\mathrm{V}=10$ under typical observing conditions $(\mathrm{V}=11$ with excellent seeing).

\subsection{BASELINE METROLOGY SYSTEM}

For the AOI to achieve its design goal of milliarcsecond astrometry, a baseline laser metrology system must be employed that can measure the three-dimensional motions of the baselines to an accuracy of approximately $0.1 \mu \mathrm{m}$ (Elias 1994). This system consists of a number of laser interferometers tied to four tempera- 
ture-stabilized super-Invar reference plates, one next to each siderostat. Five laser interferometers measure the position of a "cat's-eye" retroreflector (Danchi et al. 1986) at the intersection of the rotation axes of each siderostat, relative to the adjacent reference plate. The rotation and tilt of the reference plates will, in turn, be measured by two other metrology subsystems. In the first case, six laser interferometers will monitor changes in the distances of three points on each plate from retroreflectors embedded in a deep subsurface layer. The second subsystem consists of interferometers along lines of sight between the reference plates that detect motions of the plates in the horizontal plane. Most of the length of each laser interferometer line of site is in vacuum. Corrections are applied to the laser metrology data for changes in the optical path length due to variations of the temperature of the transmissive optics (and the variations in temperature, pressure, and relative humidity of the small remaining air paths).

Together, these various subsystems contain 56 laser interferometers that will allow a continuous measurement of the time evolution of the baselines with respect to an Earth-fixed reference system with submicron error.

Changes in the delay zero-point offset on each baseline will also be continuously monitored by infrared laser metrology beams that traverse the same optical path through the instrument as the stellar beams. The absolute value of the delay-offset will be periodically measured by fringe tracking on an internal white-light source, in a manner similar to that employed on the Mark III (Hummel et al. 1994).

\subsection{DELAY LINES}

The AOI shares with the BOA six delay lines, each providing up to $35 \mathrm{~m}$ of optical delay. The prototype of these delay lines was developed under contract at the Jet Propulsion Laboratory. These delay lines are similar to those used with the Mark III interferometer, but have smoother support and drive mechanisms that allow operation at the higher speeds and greater range of motion required by the AOI. As in the Mark III, the delay lines are entirely in vacuum to permit the wide observational bandwidth necessary for astrometric observations.

Within each delay line vacuum tank is a pair of precision rails upon which rolls the retroreflector cart. The retroreflector consists of a parabolic mirror with a piezoelectrically driven flat secondary mirror at the focus. Starlight enters the retroreflector in a beam parallel to, but above, the axis of the paraboloid, and exits in a parallel beam below the axis. The retroreflector is held in a flexible parallelogram frame on the cart; its position on the cart is adjusted by a voice coil. The cart itself is moved along the rails by a stepper motor on an auxiliary cart, which is coupled to the retroreflector cart by a second voice coil. The 
position of the retroreflector is continuously monitored by a laser metrology system. Corrections to all three positions - the cart position along the rails, the retroreflector position on the cart, and the position of the secondary mirror with respect to the primary - are generated based on the position of the central interference fringe. These delay lines can actively fringe track at typical sidereal fringe motion rates with a measured RMS smoothness of $10 \mathrm{~nm}$.

In addition to introducing the correct delays, the delay lines also introduce modulations in the delays. In the initial, three-delay-line system, one-wavelength amplitude, $500 \mathrm{~Hz}$ triangle-wave modulations are introduced in the positions of two of the three piezo-mounted secondaries. In the six-delay-line system, multiwavelength modulations at $5 \mathrm{kHz}$ will be needed to deconvolve the several baselines from each output of the beam combiner.

\subsection{BEAM COMBINER}

Unlike the Mark III, which was limited to tracking stellar fringes on only a single baseline at a time, the AOI will be capable of simultaneous observations on all its six available baselines. This is made possible by the larger number of delay lines employed, and by significantly improved beam combination and fringe detection systems.

After exiting the delay lines, the starlight beams from the several siderostats enter the beam combiner. The prototype beam combiner for the AOI and BOA produces three pairwise combinations of the input beams from three siderostats. The beam combiner will be upgraded by early 1995 to allow multi-way combination of light from as many as six siderostats for both astrometric and imaging observations (Mozurkewich 1994). With six input beams, each output of this combiner will have light from four siderostats. The fringe patterns on each baseline will be distinguished by modulating each delay line at a unique frequency.

\subsection{FRINGE DETECTION}

The output beams from the beam combiner are each dispersed in a spectrometer onto a linear array of 32 optical fibers that carry the light to each of 32 avalanche photo-diodes (APDs). The intensities in the spectral channels (spanning a wavelength range of 450 to $900 \mathrm{~nm}$ ) are detected synchronously with the delay line modulations. The result for each baseline is a two-dimensional array of photon count data, where one dimension is channel number and the other is the delay difference between the two input beams forming that baseline. A two-dimensional Fourier transform of this array yields the delay error. 
Although the beam combiner and fringe-sensor systems were originally conceived for imaging work with the BOA, they offer significant advantages for astrometric observations over the techniques employed on the Mark III. In addition to the multi-way beam combiner allowing simultaneous observations on all the available baselines, the dispersed fringe detection technique permits unambiguous central fringe identification, and better correction for atmospherically induced delay fluctuations.

\section{Milestones}

Construction at the interferometer site on Anderson Mesa began in September 1992 and the first phase (including all the infrastructure for the AOI) is now nearly complete (White et al. 1994). Access roads and the interferometer control building were completed in the spring of 1993, the optical laboratory in July, the array piers in December, and the siderostat shelters in January 1994.

The installation of those subsystems of the AOI necessary for first light was completed in August 1994. This work included the installation of the first three siderostats and the assembly and vacuum testing of the feed system. The motor and retroreflector carts were installed in the first three delay lines and tested. The beam-combiner hardware and the fringe detection electronics were also assembled and tested. The hardware and software of the interferometer control system are now essentially complete.

First light on the AOI (tracking stellar fringes on one baseline) occurred on October 28, 1994. Three-element operation is expected early in 1995. The baseline metrology system will be fully operational in mid-1995; routine astrometric observations with four siderostats will then commence.

\section{Conclusion}

The AOI, with a capability of milliarcsecond astrometry and a planned operational lifetime of several decades, will be capable of maintaining the optical reference frame by improving the proper motions of thousands of the brighter HIPPARCOS stars through repeated observations. 


\section{References}

Amstrong, J.T. (1994) Progress on the Big Optical Array (BOA), in Amplitude and Intensity Spatial Interferometry II, ed. J.B. Breckinridge, Proc. SPIE, 2200, 62.

Danchi, W.C., Arthur, A., Fulton, R., Peck, M., Sadoulet, B., Sutton, E.C., Townes, C.H., and Weitzmann, R.H. (1986) A High Precision Telescope Pointing System, in Advanced Technology Optical Telescopes III, ed. L.D. Barr, Proc. SPIE, 628, 422.

Elias, N.M. (1994) Baseline Metrology System of the USNO Astrometric Interferometer, in Amplitude and Intensity Spatial Interferometry II, ed. J.B. Breckinridge, Proc SPIE, $2200,71$.

Hummel, C.A., Mozurkewich, D., Elias, N.M., Quirrenbach, A., Buscher, D.F., Armstrong, J.T., Johnston, K.J., Simon, R.S., and Hutter, D.J. (1994) Four Years of Astrometric Measurements with the Mark III Optical Interferometer, Astron. J., 108, 326.

Mozurkewich, D. (1994) A Hybrid Design for a Six Way Beam Combiner, in Amplitude and Intensity Spatial Interferometry II, ed. J.B. Breckinridge, Proc. SPIE, $2200,76$.

Shao, M., Colavita, M.M., Hines, B.E., Staelin, D.H., Hutter, D.J., Johnston, K.J., Mozurkewich, D., Simon, R.S., Hershey, J.L., Hughes, J.A., and Kaplan, G.H. (1988) The Mark III Stellar Interferometer, Astron. Astrophys., 193, 357.

White, N.M., Millis, R.L., Franz, O.G., Loven, J.M., Hutter, D.J., Johnston, K.J., Armstrong, J.T., and Mozurkewich, D. (1994) Progress Report on the Construction of the Navy Prototype Optical Interferometer at the Lowell Observatory, in Amplitude and Intensity Spatial Interferometry II, ed. J.B. Breckinridge, Proc. SPIE, $2200,242$. 